\title{
Simulation Evaluation of Controller-Managed Spacing Tools under Realistic Operational Conditions
}

\author{
Todd J. Callantine \\ San Jose State University/ \\ NASA Ames Research Center \\ Moffett Field, CA 94035 USA \\ +16506042631 \\ todd.callantine@nasa.gov
}

\author{
Sarah M. Hunt \\ San Jose State University/ \\ NASA Ames Research Center \\ Moffett Field, CA 94035 USA \\ $+16506041695$ \\ sarah.m.hunt@nasa.gov
}

\author{
Thomas Prevot \\ NASA Ames Research Center \\ Moffett Field, CA 94035 \\ $+16506042661$ \\ thomas.prevot@nasa.gov
}

\begin{abstract}
Controller-Managed Spacing (CMS) tools have been developed to aid air traffic controllers in managing high volumes of arriving aircraft according to a schedule while enabling them to fly efficient descent profiles. The CMS tools are undergoing refinement in preparation for field demonstration as part of NASA's Air Traffic Management (ATM) Technology Demonstration-1 (ATD-1). Systemlevel ATD-1 simulations have been conducted to quantify expected efficiency and capacity gains under realistic operational conditions. This paper presents simulation results with a focus on CMS-tool human factors. The results suggest experienced controllers new to the tools find them acceptable and can use them effectively in ATD-1 operations.
\end{abstract}

\section{Keywords}

automation, controller tools, scheduling, spacing, terminal area, technology demonstration

\section{INTRODUCTION}

Streamlining arrival management to enable sustained high throughput, while reducing fuel consumption, emissions, and noise, is a cornerstone of future air transportation initiatives. Today, Area Navigation (RNAV) arrival routes go unused because current 'step-down' control techniques for ensuring safe separation interrupt them; blanket traffic management initiatives such as miles-in-trail restrictions increase controller workload and reduce capacity unnecessarily; and a lack of arrival-flow coordination forces terminal-area controllers to merge aircraft using highly inefficient low-altitude vectoring.

The FAA's Next Generation Air Transportation System (NextGen) emphasizes Performance-Based Navigation (PBN) approaches to reducing delays and inefficiencies, including scheduling arrivals to perform de-conflicted Continuous Descent Operations (CDOs) along published RNAV routes, and using Automatic Dependent

Permission to make digital or hard copies of all or part of this work for personal or classroom use is granted without fee provided that copies are not made or distributed for profit or commercial advantage and that copies bear this notice and the full citation on the first page. To copy otherwise, or republish, to post on servers or to redistribute to lists, requires prior specific permission and/or a fee.

HCI-Aero 2014, July 30-August 1, 2014, Santa Clara, California, USA

Copyright 2014 ACM 978-1-60558-246-7/09/04...\$5.00.
Surveillance-Broadcast (ADS-B) to enable Flight-Deck Interval Management (FIM) for increased inter-arrival precision [1]. If benefits are to be realized in the near- to mid-term, however, human air traffic controllers must be carefully integrated into the new ATM system.

Maintaining uninterrupted CDOs in high-traffic conditions is infeasible without suitable tools to help controllers sequence and space aircraft using speed control. The CMS tools have been developed to aid controllers in issuing speed instructions to enable aircraft to conduct efficient RNAV descents through busy terminal areas and meet scheduled arrival times. The CMS tools use schedule information and trajectory predictions along each aircraft's RNAV route to its assigned runway to provide controllers with information about the planned arrival sequence, scheduled arrival times, progress relative to the schedule, and advised speeds.

The CMS tools were initially prototyped within the Multi Aircraft Control System (MACS) simulation platform in the Airspace Operations Laboratory (AOL) at NASA Ames Research Center. Human-in-the-loop simulations were conducted to evaluate the use and effectiveness of the prototype tools and other display enhancements, along with procedures for managing high-density arrival flows with sustained high throughput and coping with disturbances and off-nominal events $[5,6,3]$.

In 2011 efforts shifted toward integrating the CMS tools with NASA-developed scheduling automation and FIM avionics as part of ATD-1. A series of simulations were conducted to support iterative refinement of an integrated prototype system and supporting concept of operations in preparation for eventual field demonstration [4]. Each integration simulation afforded the opportunity to collect data using the latest prototype, gather feedback from pilot and controller participants, and refine the CMS tools, procedures, and phraseology.

This paper presents results on CMS-tool human factors drawn from ATD-1 simulations conducted in July and September 2013 to quantify expected efficiency and capacity gains under the most realistic operational conditions possible. The simulations used a mature ATD-1 prototype that included inter-process communication protocols that meet requirements for operation in the field, 


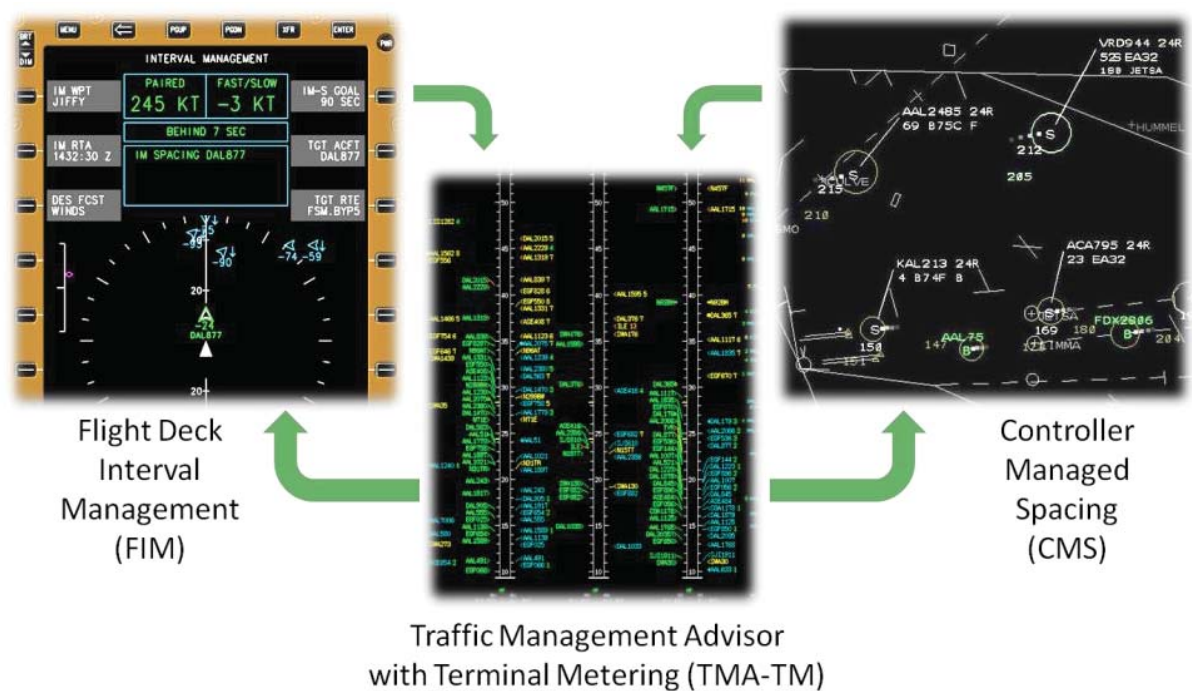

Figure 1. ATD-1 core technologies.

and a system architecture in which the CMS tools are computed by the scheduling automation and communicated to terminal-area controller workstations.

The paper first provides background on ATD-1 and the supporting operational concept. It then describes the CMS tools and the results of prior ATD-1 simulations. Following a detailed overview of the recent human-in-the-loop simulations, the paper presents results on the achieved efficiency and capacity, as well as the acceptability, usability, and usefulness of the CMS tools in their current implementation. The paper concludes with a discussion of the results and outstanding issues.

\section{BACKGROUND}

NASA initiated ATD-1 to demonstrate increased, more consistent use of PBN, demonstrate an ADS-B-In spacing application, and accelerate the transfer of NASA scheduling and spacing technologies for operational deployment. ATD-1 is a multi-year collaborative effort between researchers at NASA Ames and Langley Research Centers, the FAA, and industry partners to integrate, mature, and operationally demonstrate NASA-developed technologies that have attained a sufficient level of maturity to merit in-depth, system-level research in relevant environments [7].

ATD-1 integrates scheduling automation, CMS tools, and ADS-B-enabled FIM avionics (Fig. 1). The scheduling system is the Traffic Management Advisor for Terminal Metering (TMA-TM) developed at NASA Ames. The TMA-TM represents a significant advancement from the currently deployed TMA automation system originally developed as part of NASA's Center-TRACON Automation System (CTAS) [8]. To construct arrival schedules tailored for high-throughput CDO operations, TMA-TM generates trajectory predictions along terminalarea RNAV routes and uses them, rather than simple transit-time estimates, to generate schedules that are deconflicted at each terminal-area merge point [7].
FIM capabilities are implemented using the Airborne Spacing for Terminal Arrival Routes (ASTAR) algorithm developed at NASA Langley. ASTAR uses ADS-B data to provide flight crews speed commands, enabling aircraft to precisely achieve an assigned spacing interval behind a target aircraft at a specified 'achieveby' point. FIM operations are expected to reduce terminal-area controller workload and contribute additional inter-arrival precision for increasing the proportion of uninterrupted CDOs achievable with high throughput.

\section{ATD-1 Operational Concept}

Although ATD-1 has a distinct terminal-area focus, operations begin when the TMA-TM acquires each aircraft while it is still in cruise. TMA-TM assigns aircraft a runway, and computes estimated times-of-arrival (ETAs) at the meter fix, runway, and at intervening metering points (e.g., terminal-area fixes where RNAV routes merge). It then uses the ETAs together with required spacing information to assign scheduled times-of-arrival (STAs) at each scheduling point. When an aircraft reaches a 'freeze horizon' specified at a site-specific distance (e.g., $200 \mathrm{nmi}$ ) from the terminal area, the TMA-TM locks in its STA to provide a stable control target. En-route controllers now begin working to 'precondition' the aircraft using vectoring or other techniques as necessary to reduce the delay required to keep the aircraft within the speed control margin. Ongoing FAA en-route modernization efforts are expected to include Ground-based Interval Management (GIM) speed advisories to assist controllers in preconditioning aircraft efficiently. Following preconditioning, en-route controllers reestablish aircraft on RNAV routes (e.g., by clearing aircraft direct to the meter fix), and clear aircraft for CDOs. These procedures enable flight crews to use their onboard Flight Management Systems (FMSs) to fly efficient profiles from cruise until landing (or until a radar vector is required to turn the aircraft to join the approach procedure).

Once a FIM-equipped aircraft has been preconditioned and established on an RNAV OPD, the controller may issue it a FIM clearance. After the crew enters the required parameters into the onboard system (e.g., an Electronic Flight Bag (EFB)) and the target aircraft enters ADS-B range, the FIM avionics begin commanding speeds to achieve the required spacing at the achieve-by point. For non-FIM aircraft, terminal-area controllers use the CMS tools to issue speeds to ensure proper inter-arrival spacing while maintaining CDOs. Under nominal conditions, aircraft should cross the meter fix within their speed control 

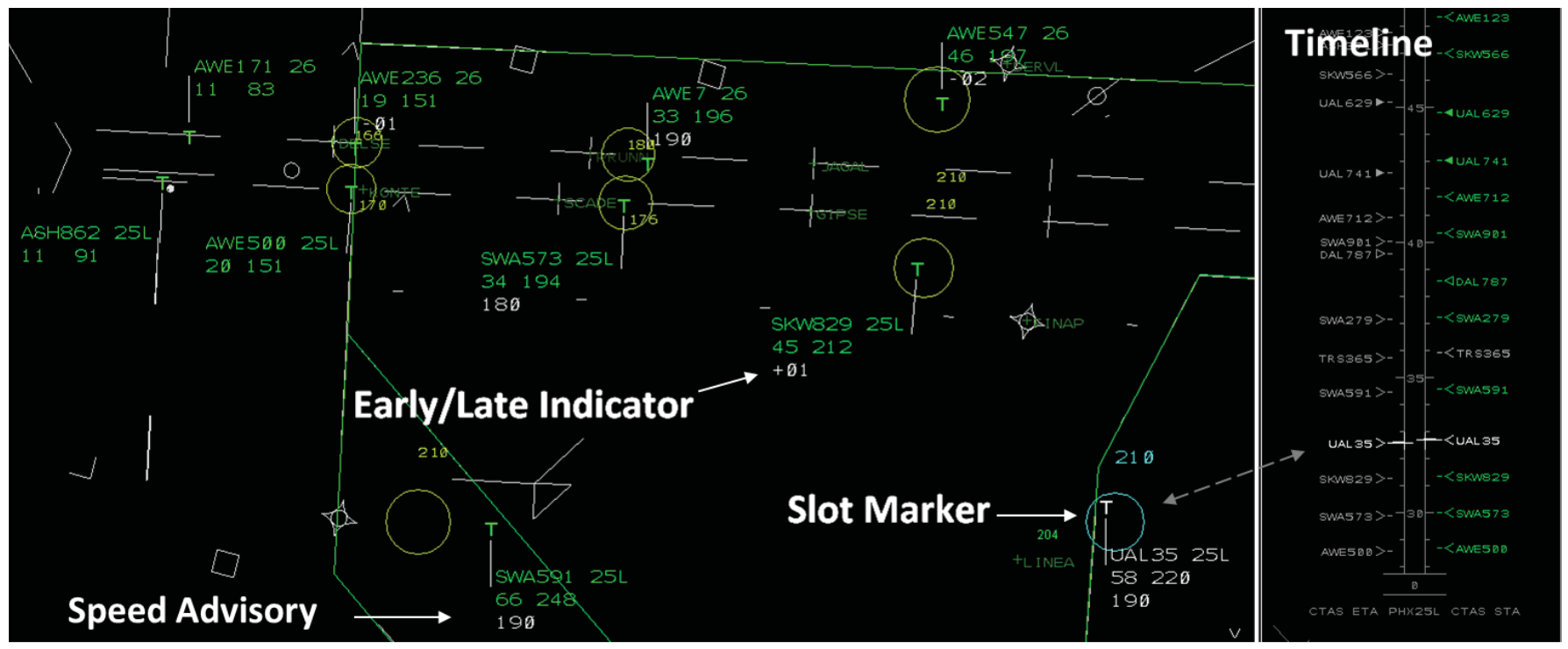

Figure 2. Controller-Managed Spacing (CMS) tools.

margin for correcting residual schedule errors and adjusting for disturbances due to winds or other factors inside the terminal area. Because controllers retain separation responsibility, they may find it necessary to interrupt CDOs or suspend FIM operations if they deem small adjustments from the nominal speed profile insufficient to maintain safe separation. All of the procedures and clearance phraseology for these operations are documented in the ATD-1 Concept of Operations [2].

\section{Controller-Managed Spacing Tools}

CMS tools are designed to help controllers manage (nonFIM) aircraft arriving on RNAV routes through the terminal area according to a schedule. Under nominal conditions, control is accomplished by adjusting the speeds of aircraft so that they can remain on efficient descent profiles along the RNAV route. CMS tools include schedule timelines, data block early/late indicators, 'slot marker' circles, and speed advisories (Fig. 2).

Timelines provide a graphical depiction of the relationship between the ETAs and STAs of aircraft crossing a specified scheduling point. This information is communicated to the controller workstation via an interface from the TMA-TM. Timelines enable controllers to assess schedule conformance by comparing an aircraft's ETA (on the left side) with its STA (on the right). If the ETA is ahead of the STA, the aircraft requires delay. Conversely, if the ETA is behind the STA, the aircraft needs to be advanced.

Timelines are part of the currently fielded TMA and have been used by traffic managers for many years. A potential problem with timelines on individual controller workstations is that they require controllers to interrupt their scan of aircraft data blocks in order to locate the desired temporal information. The ATD-1 prototype CMS tools support this process by highlighting both data blocks and timeline information for an aircraft when the controller dwells on either.
Early/late indicators add temporal information directly to the data tag. The information is more precise than the timeline information and can be gathered without taking the scan away from the traffic situation. However, it provides information only for one aircraft, rather than the overall schedule context that the timeline provides.

Controllers can use the temporal information to develop control instructions. ATD-1 is designed to use speed control along fixed paths, so speed instructions are the primary means of control under normal circumstances. CMS computes speed advisories to provide on-time performance for aircraft at the next control point. If an advised speed is available for an aircraft, it is shown in the data tag instead of an early/late indicator.

Slot markers translate the temporal schedule information into the spatial domain. Slot markers are depicted as circles on a controller's workstation, and indicate where the aircraft should be now, if it were to arrive at all scheduling points exactly on time while flying the TMA-TMcomputed speed profile used to schedule it. Slot-markerlocation computations also take into account aircraft performance characteristics and the current environmental forecast. The nominal airspeed is also indicated near the slot marker. An aircraft that is early is ahead of its slot marker; an aircraft that is late is behind its slot marker. In well-conditioned flows slot markers appear close to the aircraft-position symbol. Controllers can use the aircraft location relative to its slot marker (i.e., nominal position) and the aircraft speed relative to its nominal speed to formulate the appropriate control actions. The control task consists of putting the aircraft into its slot marker at the appropriate control points, so that separation is provided and the control problem for the downstream controller is adequately preconditioned.

This trajectory-based approach distinguishes slot markers and the CMS tools in general from other tools suggested for 
terminal-area operations. The TMA-TM uses its trajectory predictions to compute the information required to display the CMS tools and distributes it to the individual controller workstations to provide real-time spatial and temporal indications of schedule conformance as aircraft transit the terminal area.

\section{Prior ATD-1 Simulations}

Initial ATD-1 simulations in the AOL introduced controller participants experienced with CMS operations to mixedFIM-equipage arrival operations, gained initial controller acceptance, and examined a variety of controller-tool configurations (e.g., no controller tools, tools only available for non-FIM aircraft). These simulations illustrated that enroute preconditioning using precise meter-list STAs made the terminal-area controllers' task more manageable and increased the effectiveness of the CMS tools by ensuring aircraft needed only small adjustments possible with speed control. They also lead to the development of prototype FIM-status designators for controller displays.

A subsequent simulation expanded operations to two independent runways, and added emulations of GIM speed advisories to the MACS en-route controller workstations. It also refined how FIM-clearance information appeared in the en-route controllers' meter lists. Winds-at-altitude and forecast wind errors were also added. Controller participants were recently retired with an average of nearly thirty years of experience in the study airspace-but no familiarity with the CMS tools, FIM operations, or the MACS simulation platform. This afforded the opportunity to develop and evaluate controller training to support ATD1 operations. The results showed that controllers found workload low and operations acceptable, seldom vectored aircraft off the RNAV routes, rated the CMS tools consistently with prior studies, and rated the training highly. Overall, however, this study indicated a greater familiarity with the system is needed to yield consistent benefits.

Finally, a simulation conducted previous to the present studies sought to continue the systemintegration process and further investigate the effect of preconditioning arrival flows. It also sought to validate a new TMA-TM adaptation for Phoenix Sky Harbor International Airport (PHX) and integrate realistic winds with the ATD-1 laboratory prototype. It also evaluated new CMS-tool display formats for speed advisories and other ATD1-specific information. A key finding was that when en-route controllers precondition arrival flows precisely, terminal-area controllers are significantly better able to maintain efficient descents along RNAV routes while sustaining scheduled throughput.

\section{SYSTEM-LEVEL SIMULATION STUDY}

This section describes two large-scale, distributed air and ground simulations intended to quantify expected efficiency and capacity gains under realistic operational conditions when using the ATD-1 ground-based technologies. In the first simulation ('CA-5.1'), controllers worked simulated traffic using current-day workstations and control techniques. The second simulation ('CA-5.2') introduced the TMA-TM and CMS tools. The following subsections provide details on CA-5.1 and CA-5.2.

\section{Airspace and Route Structure}

The airspace surrounding PHX, including the Phoenix terminal area, and portions of Albuquerque (ZAB) and Los Angeles (ZLA) Centers were simulated. The test airspace included four terminal-area sectors, four low-altitude arrival sectors, and four high-altitude sectors (Figs. 3 and 4). Simulated RNAV-equipped aircraft flew along preexisting PHX RNAV arrival routes; unequipped aircraft flew standard arrival routes. Both west-flow (Fig. 4) and east-flow operations were simulated.

\section{Traffic Scenarios and Winds}

Traffic scenarios were developed based on actual PHX traffic samples drawn from peak arrival periods in 2011. One east-flow and one west-flow traffic sample were selected during which significant arrival rushes occurred across three of the four terminal-area entry points ('meter fixes'). Developers carefully maintained initialization times and positions and assigned aircraft to the appropriate arrival routes. Departures and over-flights were also included; these aircraft were adjusted to ensure any impacts to the test sectors were delayed until the traffic flows were well established following initialization. Scenarios were

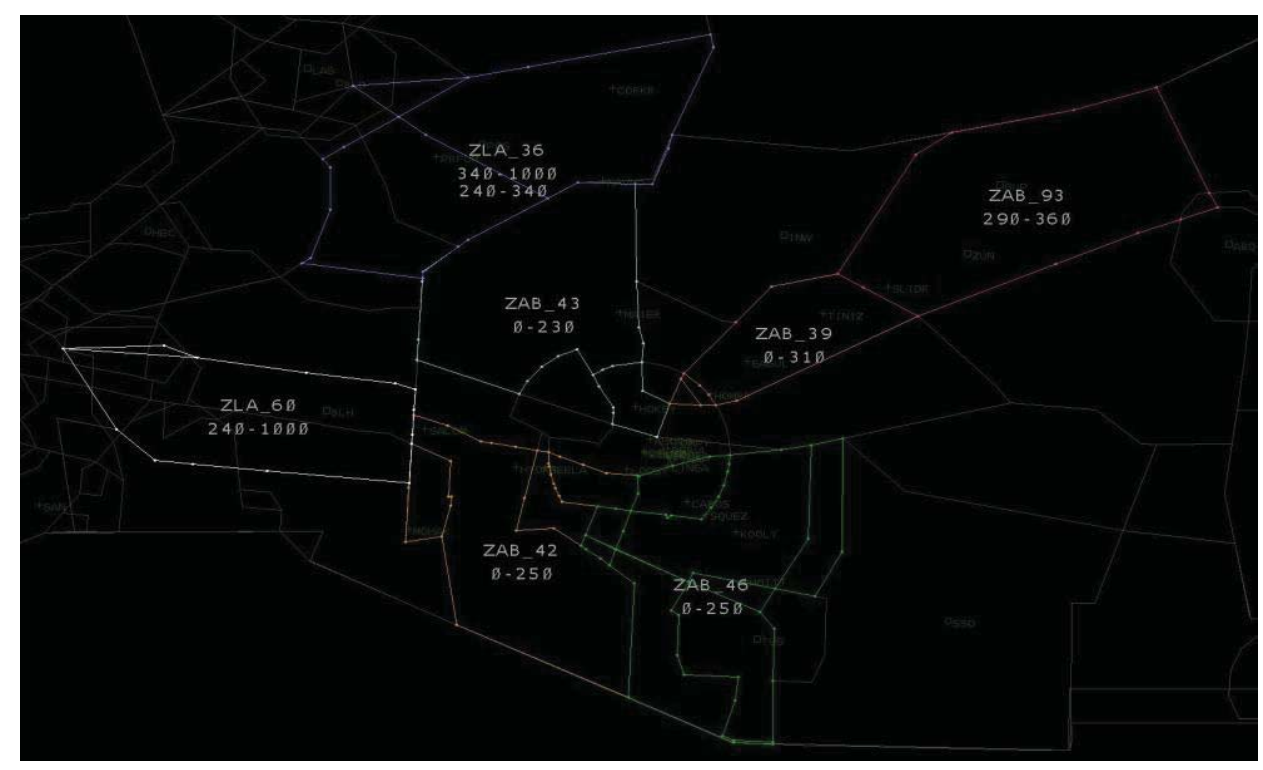

Figure 3. High- and low-altitude sectors in Center airspace 


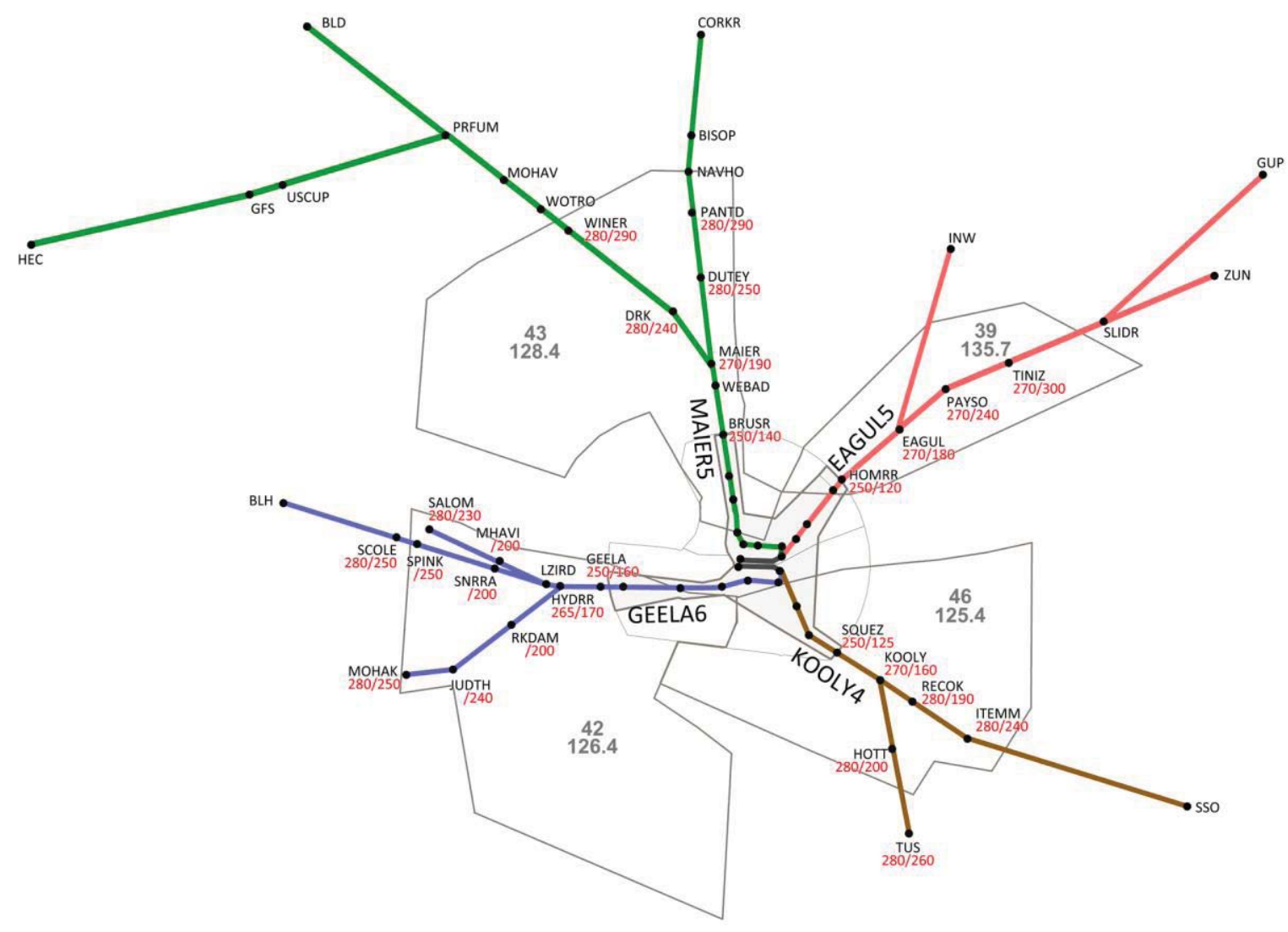

Figure 4. PHX west-flow RNAV arrival routes and arrival transitions through low-altitude en-route sectors.

designed with one-hour duration.

Environmental winds were also drawn from recorded samples chosen based on an extensive analysis; each traffic scenario was paired with four different sets of gridded winds that represent the most prevalent PHX wind patterns, as well as winds of different strengths, and crosswinds on final approach. The environmental winds were then paired with forecast winds for use in the ground-system computations according to a process that ensured a nominal overall level of wind-forecast error. Aircraft were also identified for replacement with single-pilot desktop simulators (which could perform FIM operations in a subsequent 'full ATD-1' simulation). In the end, sixteen traffic scenarios were created for the simulation: four eastflow and four west-flow scenarios drawn directly from the recorded traffic (with aircraft call-signs randomizeddenoted E01 through E04 and W01 through W04), and a second set of eight in which six aircraft were added to the original east-flow scenarios and five aircraft were added to original west-flow scenarios to provide a slightly higher traffic load (denoted E01+ through E04+ and W01+ through W04+).

\section{Controller Workstations}

Controllers used MACS emulations of the FAA En-Route Automation Modernization (ERAM) workstations in the Center and MACS emulations of Standard Terminal Automation Replacement System (STARS) workstations in the terminal area. All functionality identified as important for realism was included in the emulations. In addition, Traffic Situation Displays (TSDs) and TMA-TM timeline displays were projected on control-room walls.

\section{Participants and Training}

Experimental subjects were recently retired PHX and ZAB controllers. Four terminal-area controllers staffed two 'Feeder' and two 'Final' positions. Center controllers staffed the four low-altitude center sectors, as well as four high-altitude sectors. Two controllers with suitable experience also served as Center and terminal-area traffic managers. Other controllers experienced in the AOL served as confederates, staffing two center sectors designed to surround the study airspace, as well as the tower and a departure sector. Pilots included eight glass-cockpit pilots flying single-pilot desktop simulators; in addition, 18 regional jet pilots and aviation students staffed pseudo-pilot positions.

Because the participants were experts in current-day PHX operations, the CA-5.1 phase of the study was preceded by three days of training during which participants received instruction primarily on MACS specifics, working with pseudo-pilots, and the AOL voice communications system. CA-5.2 began with four days of training to give participants more time to gain familiarity with ATD-1 operations, including en-route flow-conditioning ('metering') in Center airspace, and use of the CMS tools in the terminal area. 


\section{Data Collection}

Four days of data collection took place during both simulations during which each of the sixteen traffic scenarios was run once, with three trials available as spares. All MACS stations and desktop flight simulators logged digital data (as did the data-communication hubs), including flight state information, pilot and controller entries, and schedule information. The TMA-TM also logged digital data. Controllers completed short questionnaires between trials, and a longer questionnaire at the end of the week. In addition, screen-capture movies that include recorded audio were collected from all MACS and ASTOR stations, and experts with extensive experience on TMA-TM and PHX operations served as observers.

\section{RESULTS}

The results of the simulation show a consistent effect of traffic-flow direction due to the selected scenarios: the eastflow traffic was more orderly, with far fewer unscheduled terminal en-route aircraft than were present in the westflow scenarios. This section first presents some systemlevel performance metrics. It then focuses on results pertinent to the use of the CMS tools in a simulated realistic arrival-management setting.

\section{Efficiency}

Efficiency is measured by the percentage of uninterrupted CDOs, referred to as PBN success rate. A successful CDO occurs when aircraft are allowed to remain on the RNAV arrival routes until they must be vectored to join the final approach. As shown in Fig. 5, PBN success rates of over $70 \%$ were achieved in CA-5.2 when the ATD-1 ground tools were in use, sometimes dramatically exceeding the PBN success rates for the CA-5.1 baseline simulation. In addition, estimates derived from recorded pilot actions indicate that, on average, terminal-area controllers issued fewer clearances per aircraft in CA-5.2.

Another measure of efficiency, flight time below 10,000 ft (considering only jet aircraft) highlights the traffic-flow differences and variability across aircraft (Fig. 6). When terminal-area controllers work without an arrival schedule, they may be able to short-cut aircraft and run aircraft in at higher speed; however, in some cases, vectoring contributes to large increases in flight time.

\section{Capacity}

Spacing accuracy on final approach (i.e., how closely aircraft conform to the required wake-vortex spacing minima) is a key determinant of achievable capacity. Fig. 7 indicates that controllers were able to reduce the standard deviation of inter-arrival spacing error at the runway threshold to some degree in all but two scenarios.

\section{Controller Acceptability}

The Controller Acceptance Rating Scale (CARS) was used to measure controller acceptability. For both CA-5.1 and CA-5.2 CARS was measured in the middle of data collection (post-Run 10). A paired samples t-test found no statistically significant difference between CA5.1 $(M=8.58)$ and CA5.2 $(M=8.17), \quad t(11)=0.861, p=.408$. The

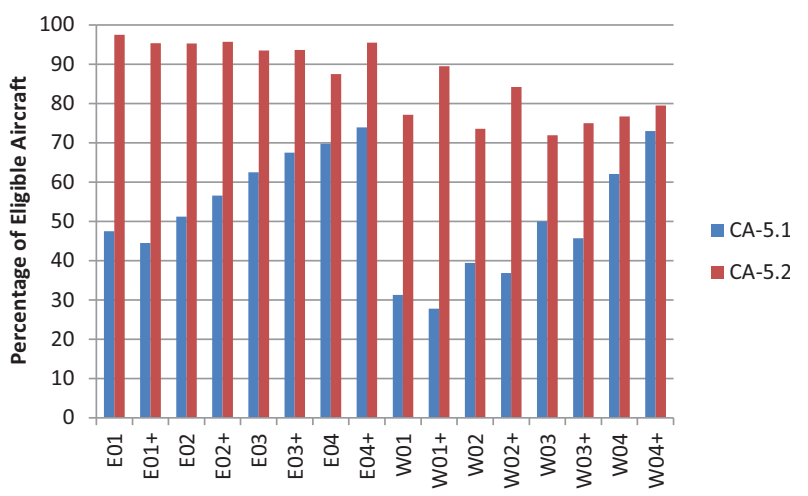

Figure 5. PBN success rate (percentage of uninterrupted CDOs) for CA-5.1 and CA-5.2.

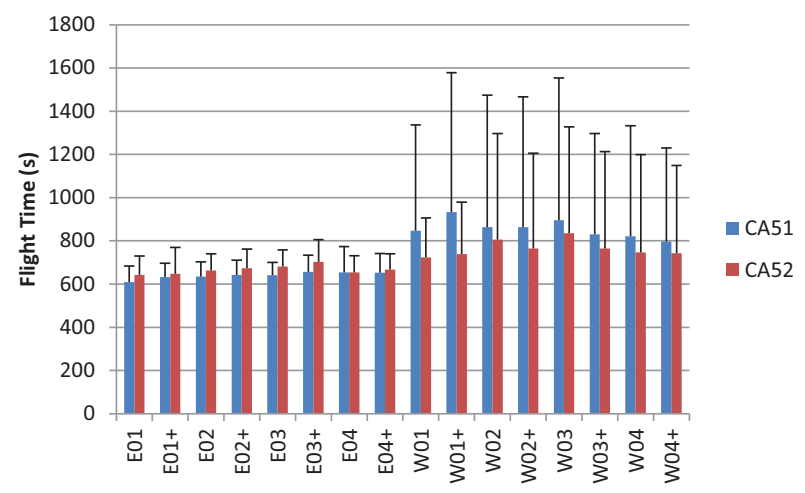

Figure 6. Flight time below 10,000 ft for CA-5.1 and CA5.2 (jet aircraft only).

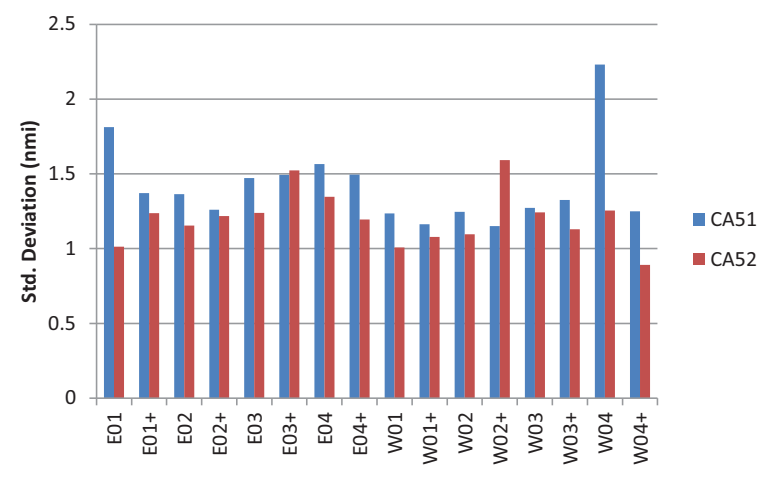

Figure 7. Standard deviation of inter-arrival spacing error for CA-5.1 and CA-5.2.

difference in sample sizes between the Center and terminal area prohibited the samples being split for hypothesis testing (Fig. 8).

\section{Controller Subjective Workload Ratings}

Figure 9 shows significant increases in item means from CA-5.1 to CA-5.2 for workload as represented by NASATLX, except the Performance subscale, where no significant change was found using related samples t-tests. Two 6 X 2 X 2 Repeated Measures ANOVAS: TLX subscale * Experiment * Flow and TLX * Experiment * 


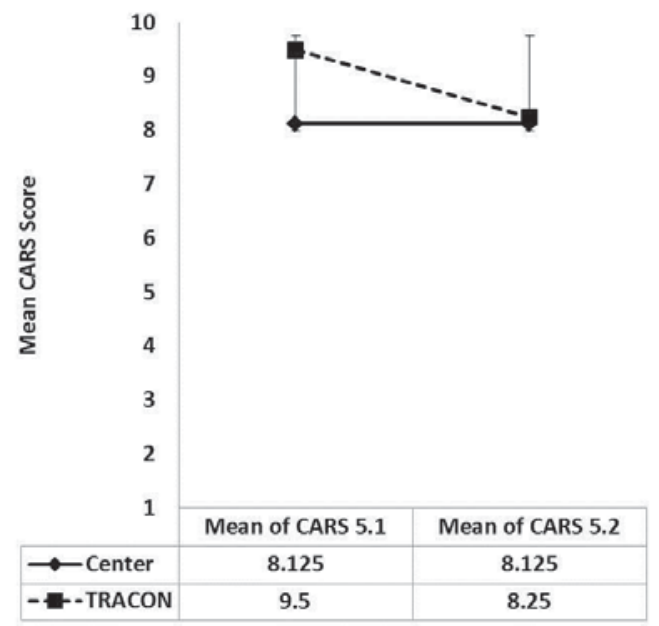

Figure 8. Mean CARS scores measured midsimulation for CA-5.1 and CA-5.2.

Traffic (Normal, Plusses) found main effects for TLX ( $F(5$, $260)=201.53, p<.001$, partial $\eta 2=7.95)$ and Experiment $(F(1,52)=89.909, p<.001$, partial $\eta 2=.634)$. Additionally, TLX subscale interacts significantly with both Experiment $(F(5,260)=23.722, p<.001$, partial $\eta 2=.313)$ (Fig. 9) and Flow $(F(5,260)=3.790, p=.025$, partial $\eta 2=.06)$ (Fig. 10), with west-flow means significantly larger than east-flow means. These results suggest that controllers who were unfamiliar with ATD-1 operations perceived CA-5.2 to demand more work than CA-5.1 without any significant change in the controllers' ratings of their performance, and that the west-flow scenarios induced higher workload than the east-flow scenarios when averaged across experiments; however, all means still remained at 'moderate load' or below, despite the significant difference. Traffic exerts a main effect when examined with TLX and Experiment $(F(1,54)=9.673, p=.023$, partial $\eta 2=.334)$, but there were no interactions between these variables.

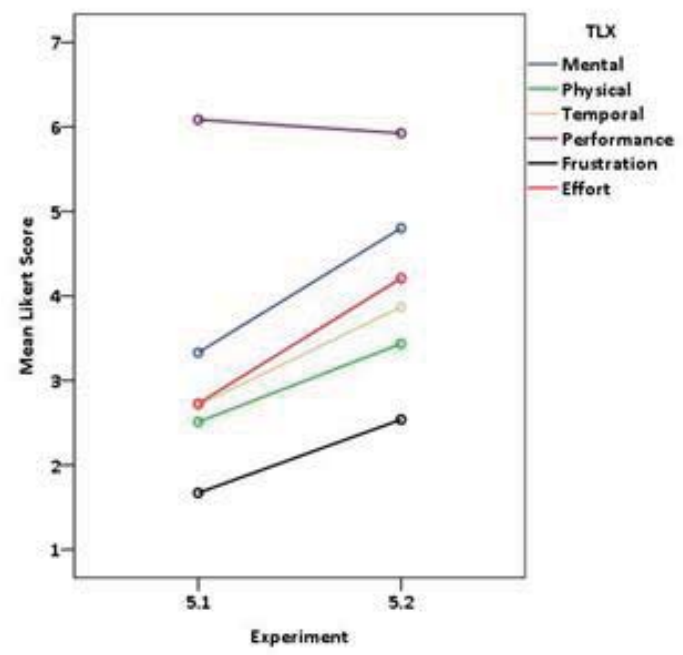

Figure 9. NASA TLX items by experiment for CA5.1 and CA-5.2.

\section{Manageability of Traffic}

Paired samples t-tests reveal significant differences in both the terminal-area and Center controllers' views of the manageability of the traffic. Terminal-area (TRACON) controllers reported their traffic to be significantly more manageable in CA-5.2 when the CMS tools were available $(M=-.426,95 \%$ CI [-.668, -.084], $t(60)=-2.494, p<.015)$, while Center controllers reported their traffic to be significantly less manageable in CA-5.2 when they were required to precondition aircraft to the schedule $(M=.707$, $95 \%$ CI $[.498, .916], t(122)=6.696, p<.001)$. However, scores remained above a value of 4 or 'quite manageable' on average despite the significant difference.

\section{CMS Tool Ratings}

For both east- and west-flow scenarios, the terminal-area controllers identified the slot markers as their most-used tool, consistent with previous CMS research. The timeline was the second most-used CMS tool. Consistent with the usage responses, slot markers were the preferred tool for spacing and speed guidance. Terminal-area controllers reported issuing speed clearances the most, followed by vectors, followed by a combination of speeds and vectors.

The timeline was mostly used as a reference to see what the automation planned. Both the speed advisories and early/late indicators were considered not nearly as helpful as the slot markers and were only used as references. Controllers reported stability issues that led them to abandon the early/late indicators. With the exception of the early/late indicators, and despite some issues reported in comments, the CMS tools were reported to be, at minimum, moderately accurate, predictable, and easy to use.

\section{CONCLUSION}

In contrast with the fielded CTAS TMA that supports delivering aircraft to the terminal area to meet a preset acceptance rate, after which controllers must rely on traditional, inefficient control techniques (i.e., vectoring) to

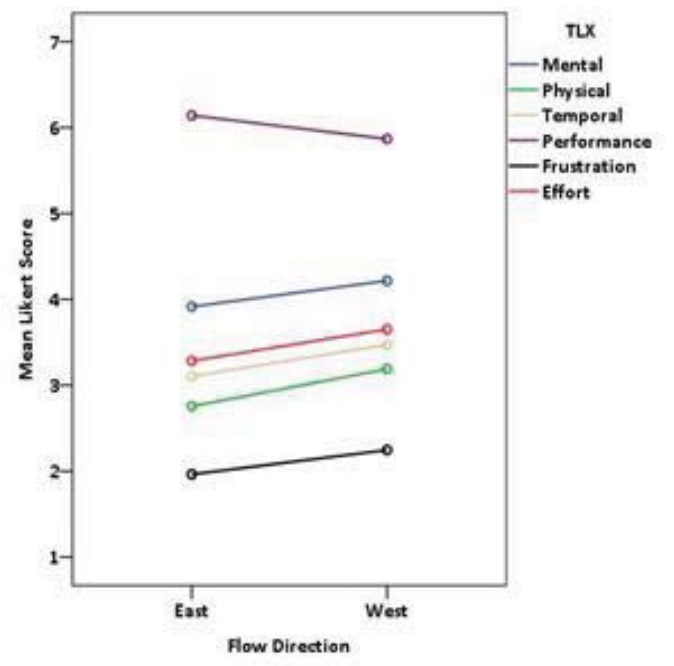

Figure 10. NASA TLX items by traffic-flow direction averaged across $\mathrm{CA}-5.1$ and $\mathrm{CA}-5.2$. 
merge and space aircraft to the runway, TMA-TM has been developed and integrated with the CMS tools under ATD-1 to further enable efficient schedule-based PBN arrivals through the terminal area. Assigning each arrival an RNAV route and landing runway early in the process helps pilots plan for an efficient descent; generating a global schedule that is de-conflicted at the meter fixes, runway, and all terminal-area merge points, and presenting the planned arrival sequence, scheduled arrival times, conformance information, and speed advisories to controllers helps them maintain efficient operations during sustained periods of high traffic.

The CA-5.1 and CA-5.2 system-level simulations suggest that ATD-1 ground-tool operations are viable in a full-scale operational environment with controllers who have received only a limited amount of training on the concept and CMS tools. While their unfamiliarity appears to have contributed to slightly lower acceptability than would be expected from experienced controllers, performance generally improved under operations with ATD-1 groundtools. CMS tool ratings again were highly consistent with those observed in prior research, with controllers finding the slot markers most usable and useful.

Moreover, follow-on work in advance of a similar systemlevel simulation of the full concept with FIM aircraft participation has resulted in an improved ATD-1 prototype in which TMA-TM-computed airspeeds match those of the aircraft more precisely, increasing the accuracy and stability of the CMS tools and the predictability of attendant control actions. In light of this, the controller performance and tool acceptability observed in CA-5.2 is particularly encouraging. In addition to testing the full ATD-1 concept, including FIM operations, the next system-level simulation will revisit CMS-tool human factors using the prototype with the corrected airspeeds.

A number of outstanding issues related to interface format and control over the CMS tools are not addressed by this research (e.g., how might controllers de-clutter their scopes in a manner suitable to certain off-nominal situations, might it be desirable to display the current ground speed of the slot marker rather than airspeed, etc.). The ATD-1 technologies are currently being transferred to the FAA for eventual deployment; low-level interface issues are expected to be addressed as part of that process.

\section{ACKNOWLEDGMENTS}

The authors would like to thank the ATD-1 project team (John Robinson, Technical Lead); AOL members Nancy Bienert, Connie Brasil, Chris Cabrall, Ashley Gomez, Vimmy Gujral, Kim Jobe, Michael Kupfer, Lynne Martin, Joey Mercer, Susan Morey, and Faisal Omar; and Harry
Swenson, Terry Smith, Liang Chen, Melody Lin, and Lee Helmle, for essential contributions to this work.

\section{REFERENCES}

1. _ FAA's NextGen Implementation Plan, Federal Aviation Administration, Washington, D.C., March 2011.

2. Baxley, B., Johnson, W., Swenson, H., Robinson, J., Prevot, T., Callantine, T., Scardina, J., and Greene, M., Air Traffic Management Technology Demonstration-1 concept of operations (ATD-1 ConOps v2.0). NASA Technical Memorandum 2013-218040. Hampton, VA: NASA Langley Research Center, September 2013.

3. Callantine, T., Cabrall, C., Kupfer, K., Martin, L., Mercer, J., and Palmer, E. (2011). Investigating the impact of off-nominal events on high-density green arrivals, in Proceedings of the 30th Digital Avionics Systems Conference, Seattle, WA, October, 2.D.5-12.D.5-15, 2011.

4. Callantine, T., Cabrall, C., Kupfer, K., Martin, L., Mercer, J., and Prevot, T., Initial investigations of controller tools and procedures for schedule-based arrival operations with mixed flight-deck interval management equipage. AIAA-2012-5673, Reston, VA: American Institute of Aeronautics and Astronautics, 2012.

5. Callantine, T., Palmer, E., and Kupfer, M., Human-inthe-loop simulation of trajectory-based terminal-area operations, in Proceedings of the 27th Congress of the International of the Aeronautical Sciences, Nice, France, September, 2010.

6. Kupfer, M., Callantine, T., Martin, L., Mercer, J., and Palmer, E., Controller support tools for schedule-based terminal operations, in Proceedings of the 9th USA/Europe Air Traffic Management Research and Development Seminar (ATM2011), Berlin, June 2011.

7. Prevot, T., Baxley, B., Callantine, T., Johnson, W., Quon, L., Robinson, J., and Swenson, H., "NASA's ATM Technology Demonstration-1: Transitioning fuel efficient, high throughput arrival operations from simulation to reality," in Proceedings of the International Conference on Human-Computer Interaction in Aerospace (HCI-Aero 2012), Brussels, September 2012.

8. Swenson, H., Hoang, T., Engelland, S., Vincent, D., Sanders, T., Sanford, B., and Heere, K. "Design and operational evaluation of the Traffic Management Advisor at the Fort Worth Air Route Traffic Control Center," in Proceedings of the $1^{\text {st }}$ USA/Europe Air Traffic Management Research and Development Seminar, Saclay, France, June 1997. 